\title{
SYNTHESIS AND CHARACTERIZATION OF A CHITOSAN-BASED CITRIC ACID-CROSSLINKED ENCAPSULANT SYSTEM
}

\author{
ANNE BERNADETTE S. AGU ${ }^{1}$, PRECIOUS JAPHETH L. BENABLO ${ }^{1}$, VINCE ST. D. MESIAS ${ }^{1}$ \\ AND DAVID P. PENALOZA JR. ${ }^{1,2 *}$
}

\author{
${ }^{I}$ Chemistry Department, College of Science, De La Salle University, 2401 Taft Avenue, Manila 1004, Philippines. \\ ${ }^{2}$ Organic Materials and Interfaces Unit, CENSER, De La Salle University, 2401 Taft Avenue, Manila 1004, Philippines.
}

\begin{abstract}
An encapsulant system was prepared via crosslinking of chitosan using citric acid as a crosslinker. FTIR spectroscopy, particle size and zeta potential analyses confirmed successful crosslinking, appropriate particle size and colloidal stability. Encapsulation capability of the said material was investigated using fluorescence spectroscopy. The effect of $\mathrm{pH}$ on the crosslinked chitosan particles was also conducted.
\end{abstract}

Keywords: Crosslinking, encapsulation, chitosan.

\section{INTRODUCTION}

Polymers have played an integral role in the delivery technology. In particular, biopolymers to devise functional materials ${ }^{1-9}$ sensitive from changes in external stimuli, e.g. temperature, pressure, moisture ${ }^{2,10-14}$ are materials of interest for smart delivery applications.

Chitosan is a polymer comprised of two glucose derivatives, D-glucosamine $(\mathrm{GlcN})$ and $\mathrm{N}$-acetyl-D-glucosamine (GlcNAc), randomly distributed and joined together by $\beta_{(1 \rightarrow 4)}$ glycosidic bonds. This natural nontoxic biopolymer is commercially produced by the partial deacetylation of chitin, a polymer composed of repeating units of GlcNAc linked by $\beta_{(1 \rightarrow 4)}$ glycosidic bonds, obtained from the exoskeleton of crustaceans and cell walls of fungi. Due to its polycationic and nontoxic nature, chitosan is extensively used and applied in the food, pharmaceuticals, and chemical industries ${ }^{3,15-16}$.

Chitosan has been used as a component in many applications in the form of hydrogels ${ }^{17-24}$, nanoparticles ${ }^{17-24}$, emulsions ${ }^{25-28}$, etc. This is due in part to its capacity to control the release of certain active agents it has encapsulated, as well as its nontoxicity and biocompatibility to the human system ${ }^{18,22,25,27,29-33}$. It can be modified using an array of methods such as crosslinking ${ }^{18,22,28,34-38}$; solution casting $^{39-42}$ and many more to produce different delivery systems, enhance absorption, and improve the relative solubility of the actives that they carry.

In this study, biopolymeric encapsulants prepared via crosslinking of chitosan were used as a delivery system. These crosslinked polymeric particles were synthesized using chitosan as an encapsulating material with citric acid as a crosslinker. This study presents a straightforward, facile preparation of a possible encapsulating system that makes use of materials that are established as nontoxic chemicals; the experimental conditions for the synthesis only require ambient temperature and the crosslinking process used water as the reaction medium. Fourier transform infrared (FTIR) and fluorescence spectroscopic methods, particle size analysis and zeta potential measurements were used to follow the crosslinking and encapsulation processes. $\mathrm{pH}$ dependency studies were also conducted by determining the stability of the esterification crosslink in acidic (pH 1.4) and above neutral ( $\mathrm{pH}$ 7.4) environments.

\section{EXPERIMENTAL}

\section{Materials}

Chitosan (high molecular weight, MW 190,000-310,000 g/mole), citric acid ( $\geq$ 99.5\%), and 8-anilino-1-naphthalenesulfonic acid ( $\geq 97 \%$ - HPLC) of analytical grade (AR) purity were purchased from Sigma Aldrich.

Preparation of crosslinked and noncrosslinked aqueous chitosan particle dispersion

Chitosan $(0.1 \mathrm{~g})$ was dispersed initially in $10 \mathrm{~mL}$ deionized water, stirred at $400 \mathrm{rpm}$. The crosslinker, citric acid at $0.1 \mathrm{~g}$ mass, was then added. The dispersion was stirred for 40 minutes at $400 \mathrm{rpm}$. The prepared crosslinked chitosan particles were then filtered using gravity filtration. A separate chitosan dispersion without the crosslinker using the same amount of materials and scheme of preparation was also followed. Each treatment solution was prepared in three replicates to establish repeatability of measurements.

\section{Characterization}

Each of the samples tested was added with $\mathrm{KBr}$ and then pelletized prior to Fourier Transform-Infrared (FTIR) analysis. The spectroscopic measurements were performed using the Thermo Scientific Class 1 Nicolet 6700 FTIR in the range of $400-4000 \mathrm{~cm}^{-1}$ at 16 scans.

The particle size and size distribution as well as the zeta potential of each formulation were determined by Malvern ZetaSizer Nano ZS instrument. The samples were spun and filtered before pipetting into the cuvettes. The measurement of particle size at a 90-degree angle was done using dynamic light scattering, while the zeta potential measurement utilized laser doppler microelectrophoresis.

The encapsulation of a model active onto the matrix was investigated by Fluorescence Spectrometer Spectrum ASCII using sodium 8-anilino-1naphthalenesulfonate (ANS) dye as a fluorophore using an excitation wavelength of $388 \mathrm{~nm}$ and an emission wavelength of $470 \mathrm{~nm}$. A $50-\mathrm{mL}$ of $5 \%(\mathrm{w} / \mathrm{v})$ dye was prepared and divided into three $15-\mathrm{mL}$ portions. The first portion contained only ANS dye, while the second and third contain $2 \mathrm{~mL}$ of uncrosslinked chitosan (without citric acid) and crosslinked chitosan (reacted with citric acid), respectively.

The morphology of the crosslinked chitosan was characterized by scanning electron microscopy using JEOL 5300 Scanning Electron Microscope.

\section{RESULTS AND DISCUSSION}

Following the crosslinking process when chitosan is reacted with citric acid

FTIR spectroscopic measurements of tested materials confirmed successful crosslinking reactions between the chitosan and the citric acid. Figure 1 shows the FTIR spectra of (a) citric acid (CA) used as the crosslinking agent; (b) chitosan used as the encapsulating material and (c) crosslinked polymer (chitosan reacted with citric acid as the crosslinking agent). As can be seen, the presence of an IR peak around $1700 \mathrm{~cm}^{-1}$ for the crosslinked chitosan (after crosslinking with CA at a weight ratio of 1:1 chitosan:CA combination). This is an indication that a crosslink has been formed from the reaction of added CA to the chitosan. The uncrosslinked chitosan (without CA) FTIR profile, in comparison, did not show any IR peak at $1700 \mathrm{~cm}^{-1}$. We also did try to adjust the chitosan-CA weight ratio (2:1), but we did not see the peak for the ester linkage. This might due to the fact that the $2: 1$ chitosan-CA weight ratio had a significantly larger amount of the free polymer, to the point that it may have possibly masked the presence of the ester linkage. Because of this, we stick with the $1: 1$ weight ratio to make the crosslinked chitosan to be further characterized. 


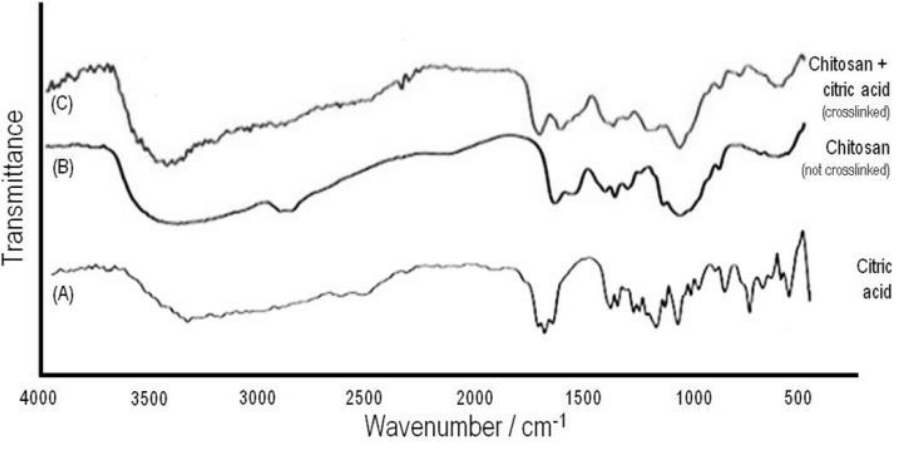

Figure 1. FTIR spectra of (A) citric acid used as the crosslinking agent; (B) chitosan used as the encapsulating material and (C) crosslinked polymer (chitosan reacted with citric acid as the crosslinking agent).

Characterizing the crosslinked chitosan in terms of size and colloidal stability in water

The size of the chitosan citric acid-crosslinked particles at a 1:1 weight ratio (chitosan-CA) was analyzed using the Malvern Zetasizer Nano ZS90 following ISO 13321. The size is determined by first measuring the Brownian motion of the particles using the dynamic light scattering. As shown in Figure 2, the size of the crosslinked particles (after crosslinking), $769 \mathrm{~nm}$, is larger than the chitosan alone, $533 \mathrm{~nm}$ (before crosslinking).
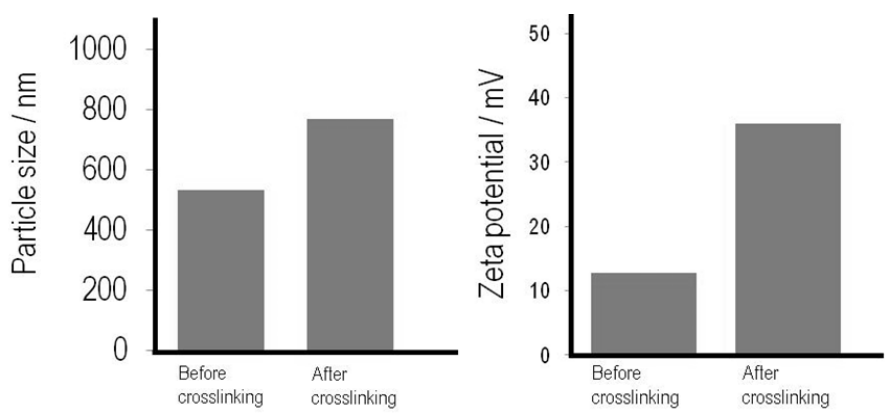

Figure 2. Particle size and zeta potential values of chitosan before and after crosslinking.

An important characteristic of polymeric particle dispersion is its colloidal stability. To check on the effect of crosslinking to the colloidal stability of the chitosan particles dispersed in water, zeta potential measurements of the chitosan before and after crosslinking were made. The zeta potential, or the electrokinetic potential, is the potential difference between the dispersive medium and the layer of fluid attached to the dispersive medium. It is also a measure of the magnitude of the electrostatic repulsion/attraction between particles. It illustrates the relative stability of nanoparticles when dispersed in water ${ }^{43}$. As shown in Figure 2 , the zeta potential value of the crosslinked particles $(+35.9 \mathrm{mV})$ is significantly higher than the uncrosslinked chitosan $(+12.7 \mathrm{mV})$ particles therefore suggesting better colloidal stability as an aqueous polymeric dispersion. At this point, what we have shown is that, crosslinked nanoparticles are larger than uncrosslinked polymers as shown by particle size measurements while zeta potential measurements revealed considerable colloidal stability of the crosslinked polymers.

\section{Does encapsulation happen?}

To investigate the capability of the formed citric acid-crosslinked chitosan nanoparticle to encapsulate an active, fluorescence spectroscopy was utilized to observe the intensity of 8-anilino-1-naphthalenesulfonate (ANS) as the fluorescent probe. The ANS dye is weakly fluorescent in an aqueous solution and provides a much stronger fluorescence emission when located in a hydrophobic environment ${ }^{44-45}$. As expected, since the ANS dye is dissolved in the aqueous solution alone in the first sample, this resulted to a lower emission intensity in the fluorescence spectra (Figure 3A). As illustrated in Figure 3C, the ANS dye dissolved in the chitosan crosslinked with citric acid solution gave the highest fluorescence intensity out of the three samples. It is worth noting the fluorescence intensity of the ANS dye contained in the aqueous dispersion is almost comparable with the aqueous ANS dye dispersion. This highlights the effect of the crosslinking of the chitosan to affect the encapsulation of the fluosrescence ANS dye as a model active. The truncation in the spectra of the citric acidcrosslinked chitosan indicates the high intensity emitted by the ANS dye in the crosslinked product. This signifies that the ANS dye was successfully trapped inside the hydrophobic domain of the citric acid-crosslinked chitosan nanoparticles. The results of the fluorescence measurements thereby confirm the encapsulation capability of the crosslinked chitosan nanoparticles.

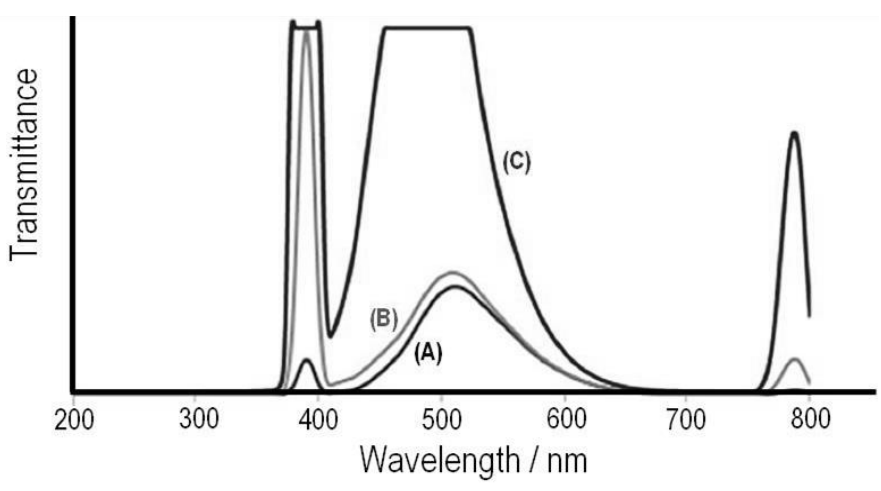

Figure 3. Fluorescence measurement of 8-Anilino-1-naphthalenesulfonic acid (ANS), a fluorescent dye, in (A) water; (B) water containing chitosan (before crosslinking) and (C) water containing chitosan and citric acid (after crosslinking). ANS dye is used as a model active to be encapsulated.

\section{Are the crosslinked particles capable of releasing actives?}

As show in Figure 4, from the presence of the distinct ester linkage, it can be said that the citric acid-crosslinked chitosan nanoparticles did not deteriorate in the extremely acidic buffer. An explanation for this could be because the nanoparticles themselves were already observed to be in a solution that was slightly acidic, which may be attributed to unreacted citric acid. Therefore, the nanoparticles retain their crosslink at acidic $\mathrm{pH}$, which can imply their potential use as an oral drug delivery system, since an influx of protons will not degrade them in any way. Interestingly enough, there was an absence of an ester linkage in the sample at $\mathrm{pH}$ 7.4. This implies that the ester crosslink does not seem to hold. Perhaps this can be explained by the possibility that the optimal $\mathrm{pH}$ for ester crosslinking is below 7, ideally at around $\mathrm{pH}$ 2.6. This can also explain the integrity of the ester linkage at $\mathrm{pH} 1.4$, since acid does not negatively affect its structure.

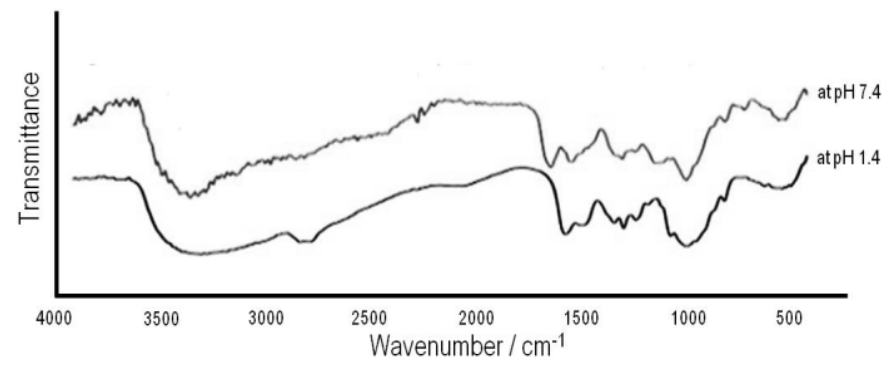

Figure 4. FTIR spectra of the crosslinked polymer (chitosan reacted with citric acid as the crosslinking agent) at $\mathrm{pH} 1.4$ and $\mathrm{pH}$ 7.4.

Further, when examined under scanning electron microscope (SEM), the morphology of the crosslinked chitosan particles exposed at these two $\mathrm{pH}$ conditions significant differs. Figure 5 shows the SEM images of the nanoparticles after having been exposed to extremely acidic (at $\mathrm{pH} 1.4$ ) and above neutral ( $\mathrm{pH}$ 7.4). The sample at $\mathrm{pH} 1.4$ has some spherical or oblongshaped structures scattered all around the imaged portion of the sample, which can be inferred as the crosslinked chitosan nanoparticles. On the other hand, the sample at $\mathrm{pH} 7.4$ shows thick fiber-like structures with relatively smooth surfaces, presumed to be the chitosan fibers alone. It is important to note, however, that this sample had larger oblong structures as compared to the previous sample. 


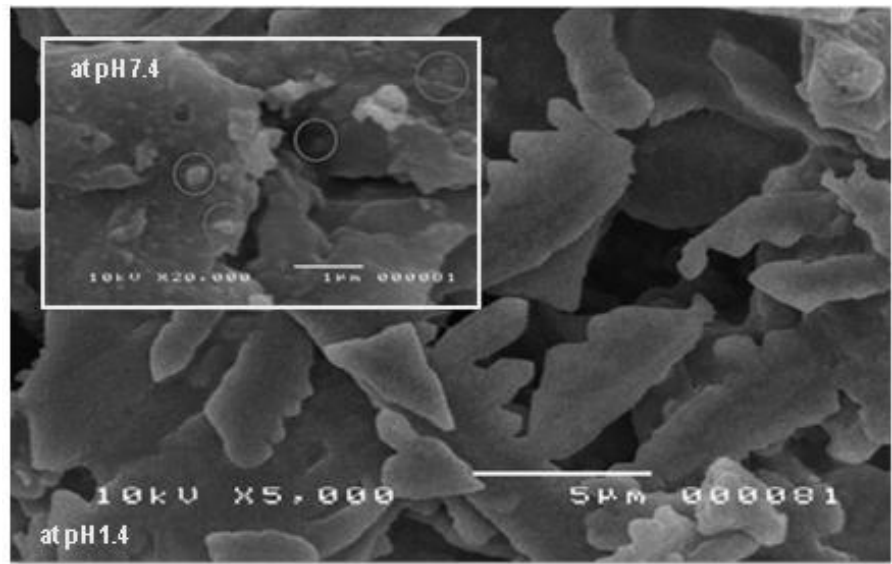

Figure 5. SEM images of the crosslinked polymer (chitosan reacted with citric acid as the crosslinking agent) at $\mathrm{pH} 1.4$ and $\mathrm{pH} 7.4$.

In summary, the FTIR spectroscopic profiles of the crosslinked particles are different with at $\mathrm{pH} 7.4$ showing the absence of the ester crosslinks. Further, SEM revealed different morphologies at these two $\mathrm{pH}$ conditions.

\section{CONCLUSION}

In conclusion, polymeric nanoparticles were synthesized using chitosan as the encapsulating matrix material and citric acid as the crosslinker. Fourier transform infrared (FTIR) spectroscopy and particle size analysis and zeta potential measurements confirmed successful crosslinking. Fluorescence spectroscopic methods established encapsulation of a model active, 8-anilino-1naphthalenesulfonic acid (ANS) by the crosslinked chitosan nanoparticles. $\mathrm{pH}$ dependence studies were also conducted and showed stability of the esterification crosslink in acidic (pH 1.4) media and degradation at relatively high $\mathrm{pH}$ (at 7.4) environments. Results from these measurements showed that chitosancrosslinked nanoparticles are promising encapsulating systems tested based on their efficient crosslinking capacity, appropriate particle size and colloidal dispersion stability, good encapsulation character, and decent stability in solution of varying $\mathrm{pH}$ environments.

\section{ACKNOWLEDGMENTS}

The authors would like to acknowledge the technical and chemical assistance extended by the D\&L Industries, Inc. and Chemrez.

\section{REFERENCES}

1. B. Balakrishnan, A. Jayakrishnan, Biomaterials 26, 3941 (2005)

2. P. Bawa, V. Pillay, Y.E. Choonara, L.C. Du Toit, Biomedical Materials 4, (2009).

3. M. Dash, F. Chiellini, R.M. Ottenbrite, E. Chiellini, Progress in Polymer Science 36, 981 (2011).

4. W.R. Gombotz, S.F. Wee, Advanced Drug Delivery Reviews 31, 267 (1998).

5. E. Khor, L.Y. Lim, Biomaterials 24, 2339, (2003).

6. G. Kogan, L. Šoltés, R. Stern, P. Gemeiner, Biotechnology Letters 29, 17 (2007).

7. D.J. McClements, E.A. Decker, Y. Park, J. Weiss, Critical Reviews in Food Science and Nutrition 49, 577 (2009).

8. W. Paul, C.P. Sharma, S.T.P. Pharma Sciences 10, 5 (2000).

9. R.A. Sperling, W.J. Parak, Philosophical Transactions of the Royal Society A: Mathematical, Physical and Engineering Sciences 368, 1333 (2010).

10. L. Altomare, L. Bonetti, C.E. Campiglio, L. De Nardo, L. Draghi, F. Tana, S. Farè, International Journal of Artificial Organs 41, 337 (2018).

11. K. Bauri, M. Nandi, P. De, Polymer Chemistry 9, 1257 (2018).

12. E. Kim, Y. Liu, H. Ben-Yoav, T.E. Winkler, K. Yan, X. Shi, J. Shen, D.L. Kelly, R. Ghodssi, W.E. Bentley, G.F. Payne, Advanced Healthcare Materials 5, 2595 (2016).

13. T. Kowalczyk, K. Hnatuszko-Konka, A. Gerszberg, A.K. Kononowicz, World Journal of Microbiology and Biotechnology 30, 2141 (2014).

14. X. Xiao, H. Zhou, K. Qian, Smart Materials and Structures 26095001 (2017).

15. C.K.S. Pillai, W. Paul, C.P. Sharma, Progress in Polymer Science 34, 641 (2009).
16. M. Rinaudo, Progress in Polymer Science 31, 603 (2006).

17. J. Berger, M. Reist, J.M. Mayer, O. Felt, R. Gurny, European Journal of Pharmaceutics and Biopharmaceutics 57, 35 (2004).

18. N. Bhattarai, H.R. Ramay, J. Gunn, F.A. Matsen, M. Zhang, Journal of Controlled Release 103, 609 (2005).

19. C.C. Lin, A.T. Metters, Advanced Drug Delivery Reviews 58, 1379 (2006).

20. G. Molinaro, J.C. Leroux, J. Damas, A. Adam, Biomaterials 23, 2717 (2002).

21. M.C.G. Pellá, M.K. Lima-Tenório, E.T. Tenório-Neto, M.R. Guilherme, E.C. Muniz, A.F. Rubira, Carbohydrate Polymers 196, 233 (2018).

22. H. Tan, C.R. Chu, K.A. Payne, K.G. Marra, Biomaterials 30, 2499 (2009).

23. M. Wang, Y. Sa, P. Li, Y. Guo, Y. Du, H. Deng, T. Jiang, Y. Wang, Materials Science and Engineering C 90, 264 (2018).

24. J. Wu, W. Wei, L.Y. Wang, Z.G. Su, G.H. Ma, Biomaterials 28, 2220 (2007).

25. P. Calvo, C. Remuñán-López, J.L. Vila-Jato, M.J. Alonso, Colloid and Polymer Science 275, 46 (1997).

26. S. Ogawa, E.A. Decker, D.J. McClements, Journal of Agricultural and Food Chemistry 51, 2806 (2003).

27. A.J. Ribeiro, C. Silva, D. Ferreira, F. Veiga, European Journal of Pharmaceutical Sciences 25, 31 (2005)

28. L.Y. Wang, G.H. Ma, Z.G. Su, Journal of Controlled Release 106, 62 (2005).

29. O. Felt, P. Buri, R. Gurny, Drug Development and Industrial Pharmacy 24, 979 (1998).

30. M. George, T.E. Abraham, Journal of Controlled Release 114, 1 (2006).

31. K.A. Janes, M.P. Fresneau, A. Marazuela, A. Fabra, M.J. Alonso, Journal of Controlled Release 73, 255 (2001).

32. A. Kumari, S.K. Yadav, S.C. Yadav, Colloids and Surfaces B: Biointerfaces $75,1(2010)$.

33. Y. Xu, Y. Du, International Journal of Pharmaceutics 250, 215 (2003).

34. J. Berger, M. Reist, J.M. Mayer, O. Felt, N.A. Peppas, R. Gurny, European Journal of Pharmaceutics and Biopharmaceutics 57, 19 (2004)

35. S.C. Chen, Y.C. Wu, F.L. Mi, Y.H. Lin, L.C. Yu, H.W. Sung, Journal of Controlled Release 96, 285 (2004).

36. M.S. Chiou, H.Y. Li, Journal of Hazardous Materials 93, 233 (2002).

37. O.A.C. Monteiro, C. Airoldi, International Journal of Biological Macromolecules 26, 119 (1999).

38. W.S. Wan Ngah, C.S. Endud, R. Mayanar, Reactive and Functional Polymers 50, 181 (2002).

39. T. Bourtoom, M.S. Chinnan, LWT - Food Science and Technology 41, 1633 (2008).

40. I. Leceta, P. Guerrero, I. Ibarburu, M.T. Dueñas, K. De La Caba, Journal of Food Engineering 116, 889 (2013).

41. T. Tanabe, N. Okitsu, A. Tachibana, K. Yamauchi, Biomaterials 23, 817 (2002).

42. K. Tomihata, Y. Ikada, Biomaterials 18, 567 (1997)

43. H.B. Fargol, Z. Yue, K.J. Ho, S. Ziqi, M. Victor, A.S. Hamed, H. Yoon-Uk, I. Masashi, D.S. Xue, Journal of the American Ceramic Society 96, 2636 (2013).

44. D.P. Penaloza Jr, A. Shundo, K. Matsumoto, M. Ohno, K. Miyaji, M. Goto, K. Tanaka, Soft Matter 9, 5166 (2013).

45. E.V. Yates, G. Meisl, T.P.J. Knowles, C.M. Dobson, The Journal of Physical Chemistry B 120, 2087 (2016). 